\title{
Semi-Analytical method for the pricing of barrier options in case of time-dependent parameters (with Matlab ${ }^{\circledR}$ codes)
}

\author{
C. Guardasoni ${ }^{1 *}$ \\ ${ }^{1}$ Department of Mathematics, Physics and Computer Science, \\ University of Parma, Italy \\ *Email address for correspondence: chiara.guardasoni@unipr.it \\ Communicated by Giorgio Fotia \\ Received on 01 12, 2017. Accepted on 02 02, 2018.
}

\begin{abstract}
A Semi-Analytical method for pricing of Barrier Options (SABO) is presented. The method is based on the foundations of Boundary Integral Methods which is recast here for the application to barrier option pricing in the Black-Scholes model with time-dependent interest rate, volatility and dividend yield. The validity of the numerical method is illustrated by several numerical examples and comparisons.

Keywords: Boundary Element Method, Black-Scholes, Barrier Options, Greeks, Time-Dependent Parameters, Integral Equations.

AMS subject classification: 65M38,91G60
\end{abstract}

\section{Introduction}

The recent financial crisis has highlighted the need for a more scientific approach to the problem of pricing and risk control, taking advantage of more advanced statistical and mathematical skills and of the availability of numerical techniques and faster computer systems.

The Black-Scholes model (BS) (see [1]) can be considered the first of the differential models for option pricing. Options are derivative contracts that give the buyer the right to buy/sell a particular asset at a predetermined price and at a fixed maturity (in case of European exercise style).

Over the past three decades, the academic literature has highlighted the strong limitations of this model due to the fact that it is based on restrictive and unrealistic assumptions: It is widely believed and experimentally verified that stocks do not have a constant spot volatility, rather this parameter varies with time... ( [2], [3], etc.). Therefore other models have been later introduced:

- models with time-dependent parameters or models where the volatility of the asset is a deterministic function of price and time; 


\section{Semi-Analytical method for the pricing of Barrier Options}

- stochastic volatility models, such as the Heston model [4], in which the value of the option depends on time, on the price and on the volatility of the underlying asset;

- jump-diffusion models, such as the Bates model [5], that involves the adoption of stochastic processes with jumps in the analysis of fluctuations of financial markets; - models assuming that log returns behave by a Levy process [6].

In the case of "barrier option", the option right gets into existence or extinguishes when the underlying asset reaches a certain barrier value. The hit of the barrier can be either discretely or continuously monitored. In the first case the benchmark methods are FFT based methods such as [6-8]. Otherwise, for continuously monitored "barrier options", pricing is traditionally based on Monte Carlo methods or on domain methods (such as Finite Element Methods and Finite Difference methods) but Monte Carlo methods are affected by high computational costs and inaccuracy due to their slow convergence and domain methods have some troubles particularly in unbounded domains.

Recently, for continuously monitored "barrier options", we have developed a stable, accurate and efficient numerical method in the context of BS with time-constant parameters [9] and for Heston and Bates models [10]. The new approach is based on the Boundary Element Method that was introduced in the Engineering field in 1970. Especially when the differential problem is defined in an unbounded domain and the data are assigned on a limited boundary (which is the case of the "barrier option"), the method is particularly advantageous for its high accuracy, for the implicit satisfaction of the far-field behavior of the solution and for the low discretization costs.

Here the method (SABO: acronym of Semi-Analytical method for the pricing of Barrier Options) is applied with some adaptations in order to solve the pricing problem of an up-and-out put option in the BS model with time-dependent parameters. Note that the explained procedure is absolutely general and it can be followed also when considering European call options and, in general, options with different single or double barriers (down/up, in/out) just resulting in different initial/boundary conditions for the starting differential problem but that can be treated in the same way. Unfortunately, on the contrary, it cannot be extended to discretely monitored barrier options.

The essential requisite for the application of the method is the knowledge of the transition probability density i.e. the fundamental solution of the differential model problem in the unbounded domain. In the here considered time-dependent BS framework, this density is known in an explicit form and, unlike the Heston and Bates contexts [10], it is easy to handle. Hence the resulting method may be easy to implement and to manage also for non-specialist finance practitioners: the only parameter that has to be evaluated is the time step of discretization. To encourage the circulation and the adoption of this method, some basic Matlab ${ }^{\circledR}$ codes described in Appendix II are downloadable as Supplemental Material of the present paper.

Barrier options are largely exchanged as they are good products for hedging and investment and they are cheaper than vanilla options. Closed formula for the pricing are available only upon satisfaction of particular hypothesis (see [11]) so, looking 


\section{Guardasoni}

at the literature, other methods can be found to price barrier options. For example: the stripe method is described in [12]; in [13] and [14] the authors provide a Fourier method but only for computing accurate closed form estimates of option price; the algorithm suggested in [15] uses a perturbative method and it is based on a series expansion, so results depends on the number of terms considered in the truncated series and on the further choice of a perturbation parameter; and so on. At last, in [16], authors apply Boundary Element Method to the problem of pricing barrier options with moving barrier and time dependent rebate but time-constant parameters. In this regard, note that the problem of pricing barrier options with time-dependent parameters and moving barrier can be brought back to the case of time-constant parameters and moving barrier but with tricky change of variables [17] that, from the numerical point of view, may be not always advantageous (the opportunity of applying change of variables to the BS partial differential problem with time-constant parameters in the vanilla options case is deeply discussed in [18]). In this sense, SABO straightforwardly applied to BS problem with time dependent parameters may be considered an alternative approach.

The outline of the paper is: in Section 2 the model problem is introduced; in Section $3 \mathrm{SABO}$ is detailed; in Section 4 there are some notes about hedging; in Section 5 other numerical methods are considered for the comparison with SABO; the obtained numerical results are collected in Section 6. Some Matlab ${ }^{\circledR}$ "ready to use" codes are downloadable at ????.

\section{The model problem}

Consider an up-and-out barrier ${ }^{\mathrm{a}}$ put option with European exercise style i.e. an exotic option that is extinguished when the price of the underlying asset grows up enough to breach an assigned upper barrier before the expiry date.

We denote by $V(S, t)$ the value of the put option dependent on the time $t$ and on the underlying asset $S$ (up to the upper barrier $S_{u}$ ), by $\sigma$ the volatility of $S$, by $E$ the exercise price, by $T$ the expiry time and by $r, d$ the interest rate and the dividend yield respectively. Differently w.r.t. the model in [9], the volatility, the interest rate and the dividend yield can be time dependent parameters, therefore the Black-Scholes problem for an European up-and-out put option reads as follows (see [19]): for $t \in\left[t_{0}, T\right), S \in\left(0, S_{u}\right)$

$$
\frac{\partial V}{\partial t}(S, t)+\frac{\sigma^{2}(t)}{2} S^{2} \frac{\partial^{2} V}{\partial S^{2}}(S, t)+(r-d)(t) S \frac{\partial V}{\partial S}(S, t)-r(t) V(S, t)=0
$$

with payoff, for $S \in\left(0, S_{u}\right)$

$$
V(S, T)=\max (E-S, 0)
$$

and boundary conditions, for $t \in\left[t_{0}, T\right]$

$$
V(0, t)=E e^{-\int_{t}^{T} r\left(t^{\prime}\right) d t^{\prime}}, \quad V\left(S_{u}, t\right)=0 .
$$

\footnotetext{
${ }^{a}$ In Appendix I, SABO is set up for the application to the case of an up-and-in put option.
} 


\section{Semi-Analytical method for the pricing of Barrier Options}

In the risk-neutral framework, performing these classical changes of variables,

$$
V(S, t)=u(x, t) e^{-\int_{t}^{T} r\left(t^{\prime}\right) d t^{\prime}}, S=e^{x}, \tau=T-t,
$$

and defining

(5)

$$
r(t)=r(T-\tau)=: \bar{r}(\tau), \quad \sigma(t)=\sigma(T-\tau)=: \bar{\sigma}(\tau), \quad \text { and } \quad d(t)=d(T-\tau)=: \bar{d}(\tau)
$$

we obtain the following diffusion problem with unknown $u(x, \tau)$

(6)

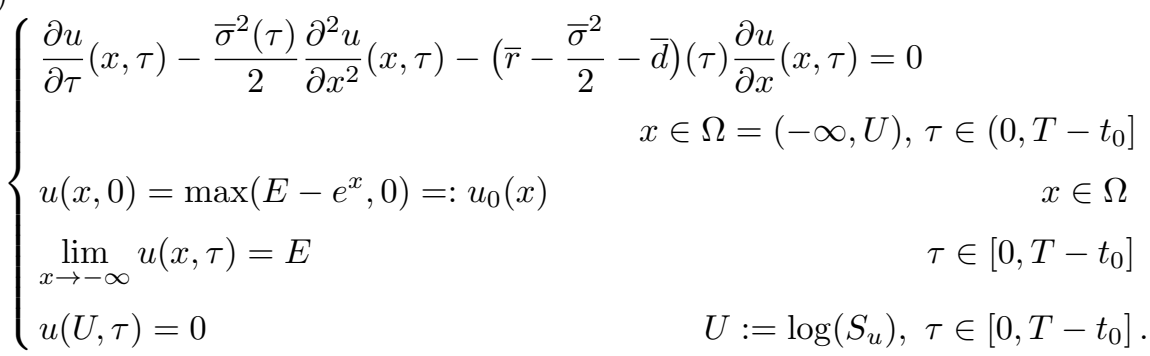

However, we remark that we could as well skip these transformations and deal with (1) directly.

Note that the exact solution of differential problem (6) is known for constant coefficients $r, \sigma, d[20]$ :

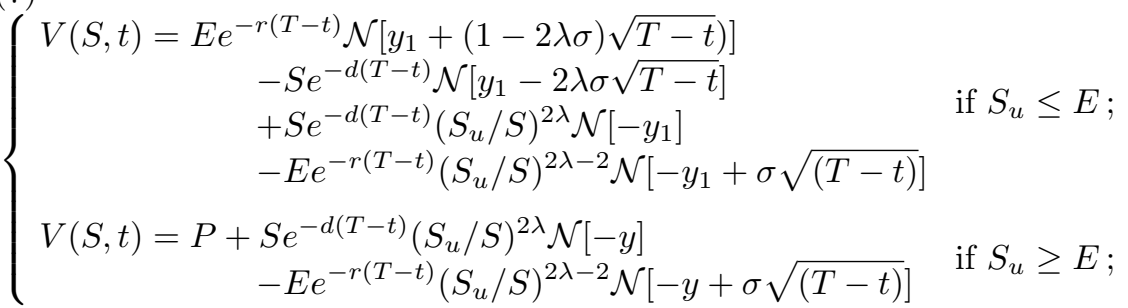

$$
\begin{aligned}
& \lambda=\frac{r-d+\sigma^{2} / 2}{\sigma^{2}} ; \quad y_{1}=\frac{\log \left(S_{u} / S\right)}{\sigma \sqrt{T-t}}+\lambda \sigma \sqrt{T-t} ; \quad y=\frac{\log \left(S_{u}^{2} /(S E)\right)}{\sigma \sqrt{T-t}}+\lambda \sigma \sqrt{T-t}
\end{aligned}
$$

where $\mathcal{N}[\cdot]$ is the normal cumulative distribution function that can be evaluated and $P(S, t)$ is the value of the European put without barriers

(8)

$$
P(S, t)=E e^{-r(T-t)} \mathcal{N}\left[-x_{1}+\sigma \sqrt{T-t}\right]-S e^{-d(T-t)} \mathcal{N}\left[-x_{1}\right] ; \quad x_{1}=\frac{\log (S / E)}{\sigma \sqrt{T-t}+\lambda \sigma \sqrt{T-t} ;}
$$

and in few other particular cases [17]. This is the reason why there is active research in numerical methods for pricing financial derivatives and in particular barrier options that are largely traded. 


\section{Guardasoni}

\section{Foundations of SABO}

Here the foundations of Boundary Element Method (BEM) are applied to the barrier option problem just illustrated in Section 2. In fact, the involved steps can be conceptually extended to the problem of barrier options pricing under general dynamics, provided that the related transition probability density is known at least in an approximate form. All these specifications of BEM can be collected in the more general method SABO.

3.1. The integral representation formula in the domain of definition of the differential problem

Let us recall some theory from [21] and [22]. Defining the operator

$$
\mathcal{L}[u](x, \tau)=\frac{\bar{\sigma}^{2}(\tau)}{2} \frac{\partial^{2} u}{\partial x^{2}}(x, \tau)+\left(\bar{r}-\frac{\bar{\sigma}^{2}}{2}-\bar{d}\right)(\tau) \frac{\partial u}{\partial x}(x, \tau)
$$

it is well known that the solution of

$$
\begin{cases}\frac{\partial u}{\partial \tau}(x, \tau)-\mathcal{L}[u](x, \tau)=0 & x \in \mathbb{R}, \tau \in\left(0, T-t_{0}\right] \\ u(x, 0)=u_{0}(x) & x \in \mathbb{R}\end{cases}
$$

is given, in an integral form, by the fundamental formula for the undiscounted price

$$
u(x, \tau):=\int_{-\infty}^{+\infty} u_{0}(y) G(y, 0 ; x, \tau) d y
$$

where

$$
G(y, s, x, \tau)=\frac{1}{\sqrt{2 \pi \int_{s}^{\tau} \bar{\sigma}^{2}(v) d v}} \exp \left\{-\frac{\left[y-x-\int_{s}^{\tau}\left(\bar{r}-\frac{\bar{\sigma}^{2}}{2}-\bar{d}\right)(v) d v\right]^{2}}{2 \int_{s}^{\tau} \bar{\sigma}^{2}(v) d v}\right\}, \quad \tau>s
$$

is the transition probability density function (PDF), also known as Green's function or fundamental solution of the partial differential problem.

For each $(x, \tau) \in \mathbb{R} \times\left[0, T-t_{0}\right)$ the $\operatorname{PDF} G(y, s ; x, \tau)$, as a function of $(y, s)$, solves the backward Kolmogorov equation

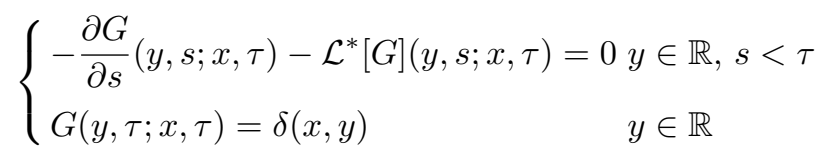




\section{Semi-Analytical method for the pricing of Barrier Options}

where $\delta(\cdot, \cdot)$ represents the Dirac distribution ${ }^{\mathrm{b}}$ and $\mathcal{L}^{*}$ is the adjoint operator of $\mathcal{L}$ defined as

$$
\mathcal{L}^{*}[\psi](y, s)=\frac{\bar{\sigma}^{2}(s)}{2} \frac{\partial^{2} \psi}{\partial y^{2}}(y, s)-\left(\bar{r}-\frac{\bar{\sigma}^{2}}{2}-\bar{d}\right)(s) \frac{\partial \psi}{\partial y}(y, s) .
$$

But, considering the differential problem (6) for an up-and-out barrier put option, the domain of investigation for $u(x, \tau)$ is $(-\infty, U) \times\left(0, T-t_{0}\right]$ and the integral formulation (10) in the new domain has to be modified inserting one more term generating the new integral representation formula

$$
u(x, \tau):=\int_{-\infty}^{U} u_{0}(y) G(y, 0 ; x, \tau) d y+\int_{0}^{\tau} \frac{\bar{\sigma}^{2}(s)}{2} G(U, s ; x, \tau) \frac{\partial u}{\partial y}(U, s) d s .
$$

In [23] a representation formula analogous to (13) is derived with a probabilistic approach together with the existence (under weak and realistic regularity hypothesis on the payoff function) of the solution of the successive Volterra integral equation (17).

Proof of validity of the representation formula (13). Let us consider the model equation

$$
\frac{\partial u}{\partial s}(y, s)-\mathcal{L}[u](y, s)=0 \quad \forall s \in(0, \tau], y \in \Omega
$$

and multiply it by the fundamental solution $G(y, s ; x, \tau)$; then subtract the PDE in (12) multiplied by $u(y, s)$ and integrate in time and space obtaining

$$
\begin{aligned}
& \int_{0}^{\tau} \int_{-\infty}^{U}\left\{G(y, s ; x, \tau) \frac{\partial u}{\partial s}(y, s)+u(y, s) \frac{\partial G}{\partial s}(y, s ; x, \tau)\right\} d y d s+ \\
& +\int_{0}^{\tau} \int_{-\infty}^{U}\left\{-G(y, s ; x, \tau) \mathcal{L}[u](y, s)+u(y, s) \mathcal{L}^{*}[G](y, s ; x, \tau)\right\} d y d s= \\
& =0 .
\end{aligned}
$$

The kernel in the second integral of (15) can be rewritten in a differential form

$$
-G(y, s ; x, \tau) \mathcal{L}[u](y, s)+u(y, s) \mathcal{L}^{*}[G](y, s ; x, \tau)=\frac{\partial p}{\partial y}(y, s)
$$

\footnotetext{
${ }^{b}$ The Dirac's delta distribution satisfies the property that $\int_{-\infty}^{+\infty} \delta(y, x) f(x) d x=$ $f(y), \forall f \in \mathcal{C}_{0}^{\infty}(\mathbb{R})$.

${ }^{c}$ The adjoint operator is defined by the condition $<\mathcal{L}(\varphi), \psi>=<\varphi, \mathcal{L}^{*}(\psi)>$ i.e., for
} any smooth functions $\psi(y)$ and $\varphi(y)$, we have

$$
\int_{-\infty}^{+\infty} \mathcal{L}[\varphi](y) \psi(y) d y=\int_{-\infty}^{+\infty} \varphi(y) \mathcal{L}^{*}[\psi](y) d y
$$




\section{Guardasoni}

with

$$
p(y, s)=\frac{\bar{\sigma}^{2}(s)}{2}\left(u \frac{\partial G}{\partial y}-G \frac{\partial u}{\partial y}\right)(y, s)-\left(\bar{r}-\frac{\bar{\sigma}^{2}}{2}-\bar{d}\right)(s)(G u)(y, s)
$$

and, integrating, we obtain

$$
\int_{0}^{\tau} \int_{-\infty}^{U}\left\{-G(y, s ; x, \tau) \mathcal{L}[u](y, s)+u(y, \tau) \mathcal{L}^{*}[G](y, s ; x, \tau)\right\} d y d s=\left.\int_{0}^{\tau} p(y, s)\right|_{y \rightarrow-\infty} ^{y=U} d s,
$$

Taking into account the natural vanishing boundary conditions for the probability density function $G$ as $y \rightarrow \pm \infty$ and the barrier condition for $u,(16)$ reduces to $^{\mathrm{d}}$

$$
\begin{aligned}
& \left.\int_{0}^{\tau} p(y, s)\right|_{y \rightarrow-\infty} ^{y=U} d s=\int_{0}^{\tau} \frac{\bar{\sigma}^{2}(s)}{2}\left(u \frac{\partial G}{\partial y}-G \frac{\partial u}{\partial y}\right)(U, s)-\left(\bar{r}-\frac{\bar{\sigma}^{2}}{2}-\bar{d}\right)(s)(G u)(U, s) d s \\
& =-\int_{0}^{\tau} \frac{\bar{\sigma}^{2}(s)}{2} G(U, s ; x, \tau) \frac{\partial u}{\partial y}(U, s) d s .
\end{aligned}
$$

The first integral in (15) can be integrated by parts

$$
\begin{aligned}
& \int_{0}^{\tau} \int_{-\infty}^{U}\left\{G(y, s ; x, \tau) \frac{\partial u}{\partial s}(y, s)+u(y, s) \frac{\partial G}{\partial s}(y, s ; x, \tau)\right\} d y d s \\
& =\int_{-\infty}^{U}\left\{\int_{0}^{\tau} G(y, s ; x, \tau) \frac{\partial u}{\partial s}(y, s) d s+\left.u(y, s) G(y, s ; x, \tau)\right|_{s=0} ^{s=\tau}-\int_{0}^{\tau} \frac{\partial u}{\partial s}(y, s) G(y, s ; x, \tau) d s\right\} d y \\
& =\int_{-\infty}^{U} u(y, \tau) G(y, \tau ; x, \tau) d y-\int_{-\infty}^{U} u(y, 0) G(y, 0 ; x, \tau) d y=
\end{aligned}
$$

and taking into account the final condition in (12), it remains

$$
=\int_{-\infty}^{U} u(y, \tau) \delta(x, y) d y-\int_{-\infty}^{U} u(y, 0) G(y, 0 ; x, \tau) d y=u(x, \tau)-\int_{-\infty}^{U} u(y, 0) G(y, 0 ; x, \tau) d y .
$$

The representation formula (13) follows immediately.

\subsection{The boundary integral equation (BIE)}

The integral formula (13) is the analytical solution of problem $(6)$ for $(x, \tau) \in$ $\Omega \times\left(0, T-t_{0}\right]$ but it cannot be evaluated analytically since the function $\frac{\partial u}{\partial y}(U, s)$ is not known explicitly.

\footnotetext{
${ }^{d}$ In the case of a plain vanilla option, taking into account that $G$ vanishes for $y \rightarrow \pm \infty$, we get the so called Green's Identity

$$
\int_{0}^{\tau} \int_{-\infty}^{+\infty}\left\{-G(y, s ; x, \tau) \mathcal{L}[u](y, s)+u(y, s) \mathcal{L}^{*}[G](y, s ; x, \tau)\right\} d y d s=0
$$

and, by substituting this into (15), we obtain the fundamental pricing formula (10).
} 


\section{Semi-Analytical method for the pricing of Barrier Options}

However, letting $x \rightarrow U$ in (13) and applying the vanishing boundary condition at the barrier, we obtain the BIE

$$
0=u(U, \tau):=\int_{-\infty}^{U} u_{0}(y) G(y, 0 ; U, \tau) d y+\int_{0}^{\tau} \frac{\bar{\sigma}^{2}(s)}{2} \frac{\partial u}{\partial y}(U, s) G(U, s ; U, \tau) d s .
$$

whose sole unknown is the function $\frac{\partial u}{\partial y}(U, s)$.

The Boundary Element Method (BEM) is based on the idea to numerically solve (17) at the boundary getting $\frac{\partial u}{\partial y}(U, s)$ and then recover the solution $u$ at the desired points of the whole domain by inserting it in the representation formula (13).

The final option price $V(S, t)$ is evaluated by transforming back with formulas (4).

\subsection{The numerical approximation of the BIE solution}

As in [9], we apply the collocation method to (17) in order to find an approximation of $\frac{\partial u}{\partial y}(U, s)$ :

- we consider a uniform decomposition of the time interval $\left[0, T-t_{0}\right]$ with time step length $\Delta t=\left(T-t_{0}\right) / N_{\Delta t}, N_{\Delta t} \in \mathbb{N}^{+}$

$$
t_{k}=k \Delta t, \quad k=0, \ldots, N_{\Delta t}
$$

- we choose temporally piecewise constant basis functions

$$
\varphi_{k}(s):=H\left[s-t_{k-1}\right]-H\left[s-t_{k}\right], \quad k=1, \ldots, N_{\Delta t},
$$

where $H[\cdot]$ denotes the Heaviside step function, for the approximation of the unknown

$$
\frac{\partial u}{\partial y}(U, s) \approx \phi(s):=\sum_{k=1}^{N_{\Delta t}} \alpha_{k} \varphi_{k}(s)
$$

- we substitute $\phi(s)$ in the boundary integral equation (17) and we evaluate it in the collocation points

$$
0=u\left(U, \bar{t}_{j}\right)=\int_{-\infty}^{U} u_{0}(y) G\left(y, 0 ; U, \bar{t}_{j}\right) d y+\int_{0}^{\bar{t}_{j}} \sum_{k=1}^{N_{\Delta t}} \alpha_{k} \varphi_{k}(s) \frac{\bar{\sigma}^{2}(s)}{2} G\left(U, s ; U, \bar{t}_{j}\right) d s
$$

choosing, as collocation points, the barycenters of intervals $\left[t_{j-1}, t_{j}\right]$, as usual when considering piecewise constant trial functions

$$
\bar{t}_{j}=\frac{t_{j}+t_{j-1}}{2}, \quad j=1, \ldots, N_{\Delta t}
$$

- we solve the resulting linear system

$$
\mathcal{A} \alpha=\mathcal{F}
$$

whose unknowns are the coefficients of (18) collected in the vector $\alpha=$ $\left(\left.\alpha_{k}\right|_{k=1, \ldots, N_{\Delta t}}\right)$. Note that, as the fundamental solution (11) is defined only for 


\section{Guardasoni}

$\tau>s, \mathcal{A}$ is a lower triangular matrix of dimension $N_{\Delta t}^{2}$

$$
\mathcal{A}=\left[\begin{array}{ccccc}
A_{11} & 0 & 0 & \cdots & 0 \\
A_{21} & A_{22} & 0 & \cdots & 0 \\
A_{31} & A_{32} & A_{33} & \cdots & 0 \\
\vdots & \cdots & \ddots & \ddots & \vdots \\
A_{N_{\Delta t} 1} & A_{N_{\Delta t} 2} & \cdots & A_{N_{\Delta t} N_{\Delta t}-1} & A_{N_{\Delta t} N_{\Delta t}}
\end{array}\right]
$$

with entries, for $j, k=1, \ldots, N_{\Delta t}, j \geq k$,

(21)

$$
\begin{aligned}
& \mathcal{A}_{j k}=\int_{0}^{\bar{t}_{j}} \varphi_{k}(s) \frac{\bar{\sigma}^{2}(s)}{2} G\left(U, s ; U, \bar{t}_{j}\right) d s=\int_{t_{k-1}}^{\min \left(t_{k}, \bar{t}_{j}\right)} \frac{\bar{\sigma}^{2}(s)}{2} G\left(U, s ; U, \bar{t}_{j}\right) d s= \\
& =\int_{t_{k-1}}^{\min \left(t_{k}, \bar{t}_{j}\right)} \frac{\bar{\sigma}^{2}(s)}{2 \sqrt{2 \pi \int_{s}^{\bar{t}_{j}} \bar{\sigma}^{2}(v) d v}} \exp \left\{-\frac{\left[\int_{s}^{\bar{t}_{j}}\left(\bar{r}-\frac{\bar{\sigma}^{2}}{2}-\bar{d}\right)(v) d v\right]^{2}}{2 \int_{s}^{\bar{t}_{j}} \bar{\sigma}^{2}(v) d v}\right\} d s
\end{aligned}
$$

and the rhs entries are

$$
\begin{aligned}
& \mathcal{F}_{j}=-\int_{-\infty}^{U} u_{0}(y) G\left(y, 0 ; U, \bar{t}_{j}\right) d y= \\
& =-\int_{-\infty}^{\min (U, \log (E))} \frac{\left(E-e^{y}\right)}{\sqrt{2 \pi \int_{0}^{\bar{t}_{j}} \bar{\sigma}^{2}(v) d v}} \exp \left\{-\frac{\left[y-U-\int_{0}^{\bar{t}_{j}}\left(\bar{r}-\frac{\bar{\sigma}^{2}}{2}-\bar{d}\right)(v) d v\right]^{2}}{2 \int_{0}^{\bar{t}_{j}} \bar{\sigma}^{2}(v) d v}\right\} d y .
\end{aligned}
$$

Note that, if $\bar{r}, \bar{\sigma}$ and $\bar{d}$ are independent of time, the matrix (20) becomes of Toeplitz type, i.e. entries are equal along the same diagonal and, consequently, the computation is faster because only the first column has to be evaluated [9].

\subsection{The numerical approximation of option price}

Once all the elements of linear system (19) have been evaluated, the approximated solution $\phi(s)$ of the BIE (17), expressed by the vector of coefficients $\alpha$ in (18), can be easily and efficiently obtained by forward substitution.

Then, as told in Section 3.2, we have to insert $\alpha$ in the representation formula (13)

$$
\begin{aligned}
& u(x, \tau) \approx \int_{-\infty}^{U} u_{0}(y) G(y, 0 ; x, \tau) d y+\sum_{k=1}^{\operatorname{ceil}\left[\frac{\tau}{\Delta t}\right]} \alpha_{k} \int_{t_{k-1}}^{\min \left(t_{k}, \tau\right)} \frac{\bar{\sigma}^{2}(s)}{2} G(U, s ; x, \tau) d s= \\
& =\int_{-\infty}^{\min (U, \log (E))} \frac{\left(E-e^{y}\right)}{\sqrt{2 \pi \int_{0}^{\tau} \bar{\sigma}^{2}(v) d v}} \exp \left\{-\frac{\left[y-x-\int_{0}^{\tau}\left(\bar{r}-\frac{\bar{\sigma}^{2}}{2}-\bar{d}\right)(v) d v\right]^{2}}{2 \int_{0}^{\tau} \bar{\sigma}^{2}(v) d v}\right\} d y+ \\
& +\sum_{k=1}^{\operatorname{ceil}\left[\frac{\tau}{\Delta t}\right]} \alpha_{k} \int_{t_{k-1}}^{\min \left(t_{k}, \tau\right)} \frac{\bar{\sigma}^{2}(s)}{2 \sqrt{2 \pi \int_{s}^{\tau} \bar{\sigma}^{2}(v) d v}} \exp \left\{-\frac{\left[U-x-\int_{s}^{\tau}\left(\bar{r}-\frac{\bar{\sigma}^{2}}{2}-\bar{d}\right)(v) d v\right]^{2}}{2 \int_{s}^{\tau} \bar{\sigma}^{2}(v) d v}\right\} d s .
\end{aligned}
$$

${ }^{e}$ ceil[[]:=function that rounds its argument to the nearest integers towards plus infinity. 


\section{Semi-Analytical method for the pricing of Barrier Options}

Finally we can get the option price applying the relation (4): $\forall S \in(0, S u), \forall t \in$ $\left[t_{0}, T\right)$

$$
V(S, t)=u(\log (S), T-t) e^{-\int_{t}^{T} r\left(t^{\prime}\right) d t^{\prime}}
$$

The main advantage of this method is that we can avoid to evaluate the solution over a grid considering only the evaluation at the points of interest.

Remarks about numerical integration

The boundary integral method typically requires the construction of ad hoc quadrature formulas to handle weak, strong and hyper singularities that appear in the boundary integral equations (see for example [24]). But, when applied to this context, only weak singularities arise and no particular quadrature rules have been implemented for the evaluation of integrals in the system entries and in the postprocessing. All the codes described in Appendix II simply exploit the adaptive quadrature functions quad and quadgk of Matlab ${ }^{\circledR}$.

However, using them, take care that:

- matrix diagonal entries (21) have a weak singularity for $s \rightarrow \bar{t}_{j}$,

- rhs entries (22) are integrals over a semi-infinite interval,

- in the approximated integral representation formula (23) both the two previous troubles appear.

In all these cases it is opportune the use of quadgk that supports infinite intervals and can handle moderate singularities at the endpoints.

Further, take care that the presence of a discontinuity in the parameters functions (i.e. the case of piecewise constant $\bar{\sigma}$ in Section 6.2) needs the application of the option Waypoints even for the numerical integration of the very simple $\int_{s}^{\tau} \bar{\sigma}^{2}(v) d v$. But, obviously, this difficulty has to be overcome also in vanilla closed formula and, in any case, it is not strictly related to barrier options in general and to this method in particular.

We might also customize strategies in order to optimize quadrature and to improve efficiency (as in [16] and [9]) but this is beyond the scope of this work that would suggest a user-friendly method that does not demand experience in determining several numerical parameters.

\section{Hedging}

Traders may want to balance their portfolios using options as risk management tools, with the help of knowledge of the associated Greeks. An option trader usually concentrates on enhancing gain or avoiding losses, on assessing risks and deciding whether they are acceptable. The knowledge of impact of Greeks on different hedging strategies will lead to determine how much risk and potential rewards are associated with the portfolio.

This section highlights how easily and straightforwardly evaluate the Greeks using $\mathrm{SABO}$, for $t \in\left[t_{0}, T\right), S \in\left(0, S_{u}\right)$

$$
\boldsymbol{\Delta}(S, t):=\frac{\partial V}{\partial S}(S, t)=\frac{1}{S} \frac{\partial u}{\partial x}(\log (S), T-t) e^{-\int_{t}^{T} r\left(t^{\prime}\right) d t^{\prime}}
$$




\section{Guardasoni}

it can be immediately obtained deriving the representation formula (13): (26)

$$
\frac{\partial u}{\partial x}(x, \tau):=\int_{-\infty}^{U} u_{0}(y) \frac{\partial G}{\partial x}(y, 0 ; x, \tau) d y+\int_{0}^{\tau} \frac{\bar{\sigma}^{2}(s)}{2} \frac{\partial G}{\partial x}(U, s ; x, \tau) \frac{\partial u}{\partial y}(U, s) d s
$$

with

$$
\frac{\partial G}{\partial x}(y, s, x, \tau)=G(y, s, x, \tau) \frac{y-x-\int_{s}^{\tau}\left(\bar{r}-\frac{\bar{\sigma}^{2}}{2}-\bar{d}\right)(v) d v}{\int_{s}^{\tau} \bar{\sigma}^{2}(v) d v} .
$$

$$
\text { - } \boldsymbol{\Gamma}(S, t):=\frac{\partial^{2} V}{\partial S^{2}}(S, t)=\frac{e^{-\int_{t}^{T} r\left(t^{\prime}\right) d t^{\prime}}}{S^{2}}\left\{\frac{\partial^{2} u}{\partial x^{2}}(\log (S), T-t)-\frac{\partial u}{\partial x}(\log (S), T-t)\right\}
$$

it can be evaluated analogously to $\Delta$, with (27) and (29)

$$
\frac{\partial^{2} u}{\partial x^{2}}(x, \tau):=\int_{-\infty}^{U} u_{0}(y) \frac{\partial^{2} G}{\partial x^{2}}(y, 0 ; x, \tau) d y+\int_{0}^{\tau} \frac{\bar{\sigma}^{2}(s)}{2} \frac{\partial^{2} G}{\partial x^{2}}(U, s ; x, \tau) \frac{\partial u}{\partial y}(U, s) d s
$$

and

$$
\frac{\partial^{2} G}{\partial x^{2}}(y, s, x, \tau)=G(y, s, x, \tau)\left\{\left[\frac{y-x-\int_{s}^{\tau}\left(\bar{r}-\frac{\bar{\sigma}^{2}}{2}-\bar{d}\right)(v) d v}{\int_{s}^{\tau} \bar{\sigma}^{2}(v) d v}\right]^{2}-\frac{1}{\int_{s}^{\tau} \bar{\sigma}^{2}(v) d v}\right\} .
$$

$$
\text { - } \Theta(S, t):=\frac{\partial V}{\partial t}(S, t)=-\frac{\partial u}{\partial \tau}(\log (S), T-t) e^{-\int_{t}^{T} \bar{r}\left(t^{\prime}\right) d t^{\prime}}+V(S, t) \bar{r}(t)
$$

for which, considering Eq. in (14) and its adjoint in (12),

$$
\frac{\partial u}{\partial \tau}(x, \tau):=\int_{-\infty}^{U} u_{0}(y) \frac{\partial G}{\partial \tau}(y, 0 ; x, \tau) d y+\int_{0}^{\tau} \frac{\bar{\sigma}^{2}(s)}{2} \frac{\partial G}{\partial \tau}(U, s ; x, \tau) \frac{\partial u}{\partial y}(U, s) d s
$$

and

$$
\begin{aligned}
\frac{\partial G}{\partial \tau}(y, s, x, \tau) & =\frac{G(y, s, x, \tau)}{\int_{s}^{\tau} \bar{\sigma}^{2}(v) d v}\left\{\left[y-x-\int_{s}^{\tau}\left(\bar{r}-\frac{\bar{\sigma}^{2}}{2}-\bar{d}\right)(v) d v\right]\left(\bar{r}-\frac{\bar{\sigma}^{2}}{2}-\bar{d}\right)(\tau)+\right. \\
& \left.+\left(\frac{\left[y-x-\int_{s}^{\tau}\left(\bar{r}-\frac{\bar{\sigma}^{2}}{2}-\bar{d}\right)(v) d v\right]^{2}}{\int_{s}^{\tau} \bar{\sigma}^{2}(v) d v}-1\right) \frac{\bar{\sigma}^{2}(\tau)}{2}\right\} .
\end{aligned}
$$

Substituting $\frac{\partial u}{\partial y}(U, s)$ by its approximation $\phi$ (known once the linear system (19) is solved) in (26), (29) and (32) we can straightforwardly compute the related Greeks.

Observe that the "secondary" unknowns $\Delta$ and $\Gamma$ can be evaluated even without computing the primary unknown $V$ but, anyway, for all the "secondary" unknowns we need at most the computation of $V$ only at the point $(S, t)$ where its derivatives are required. 


\section{Semi-Analytical method for the pricing of Barrier Options}

\section{Comments on other numerical methods}

Other numerical methods have been taken into consideration to check the efficiency of SABO. For a survey of numerical methods usually applied in pricing financial derivatives, look at [25].

The inferiority of standard Monte Carlo method w.r.t. other methods when comparing convergence speed is well known [26]. In general its order of convergence depends on $M^{-1 / 2}$ where $M$ is the number of considered samples; in this kind of problem the sampling is coupled in time with a finite difference method that makes convergence even slower and that can even introduce some bias so it is here skipped as the related results appear to be insignificant.

Therefore, in the following examples, the comparison is made with results available in literature and with results obtained by an implicit Finite Difference method. In particular, the forward Euler scheme and centered finite difference schemes have been directly applied to the backward problem (1)-(3) for the approximation of derivatives in time and in space respectively. Defining

$$
\begin{array}{ll}
\Delta S=S_{u} / N_{S}, \quad S_{i}=i \Delta S & i=0, \ldots, N_{S} \\
\Delta t=\left(T-t_{0}\right) / N_{t}, t_{i}=i \Delta t & i=0, \ldots, N_{t} \\
V_{i}^{j}:=V\left(S_{i}, t_{j}\right) \quad i=1, \ldots, N_{S}-1 j=0, \ldots, N_{t}-1 \\
\mathbf{V}_{F D}^{j} \approx \mathbf{V}^{j}=\left\{V_{i}^{j}\right\}_{i=1, \ldots, N_{S}-1}
\end{array}
$$

we have to solve the following sequence of tridiagonal linear systems: for $j=$ $N_{t}-1, \ldots, 0$

$$
\mathbf{A} \mathbf{V}_{F D}^{j}=\mathbf{B}
$$

with

$$
\begin{aligned}
& \mathbf{A}=\left[\begin{array}{ccc}
\left(1+\Delta t\left(\frac{S_{1} \sigma^{j}}{\Delta x}\right)^{2}+\Delta t r^{j}\right) & -\frac{\Delta t}{2}\left(\left(\frac{S_{1} \sigma^{j}}{\Delta x}\right)^{2}+\frac{S_{1}\left(r^{j}-d^{j}\right)}{\Delta x}\right) & \mathbf{0} \\
-\frac{\Delta t}{2}\left(\left(\frac{S_{2} \sigma^{j}}{\Delta x}\right)^{2}-\frac{S_{2}\left(r^{j}-d^{j}\right)}{\Delta x}\right) & \ldots & -\frac{\Delta t}{2}\left(\left(\frac{S_{N_{S}-2} \sigma^{j}}{\Delta x}\right)^{2}+\frac{S_{N_{S}-2}\left(r^{j}-d^{j}\right)}{\Delta x}\right) \\
\mathbf{0} & -\frac{\Delta t}{2}\left(\left(\frac{S_{N_{S}-1} \sigma^{j}}{\Delta x}\right)^{2}-\frac{S_{N_{S}-1}\left(r^{j}-d^{j}\right)}{\Delta x}\right) & \left(1+\Delta t\left(\frac{S_{N_{S}-1} \sigma^{j}}{\Delta x}\right)^{2}+\Delta t r^{j}\right)
\end{array}\right] \\
& \mathbf{B}=\left[\begin{array}{cc}
V_{1}^{j+1}+\frac{\Delta t}{2}\left(\left(\frac{S_{1} \sigma^{j}}{\Delta x}\right)^{2}-\frac{S_{1}\left(r^{j}-d^{j}\right)}{\Delta x}\right) E \exp \left(-\int_{t_{j}}^{T} r(\tau) d \tau\right), V_{2}^{j+1}, \ldots, V_{N_{S}-1}^{j+1}
\end{array}\right]^{\prime}
\end{aligned}
$$

and the upper index $j$ means that functions are evaluated at $t_{j}$.

This method (to which we briefly refer as FD) is implicit and it is expected to be of first order consistency in time and second order in space. So defining $\Delta t$ and $\Delta S$ the discretization parameters in time and space respectively, in the numerical examples, the FD time-space grid has been refined by following the relation $\Delta t=\Delta S^{2}$ with the aim to have the same order of error in both variables.

Other methods, theoretically more accurate than FD (such as CranckNicolson in time or Rannacher time stepping [27]) are not taken into consideration because they do not significantly improve numerical results in this context due to the non-smoothness of the underlying data, as described in 


\section{Guardasoni}

several papers ( [28], [29] [30] and [31]) and personally observed in [9]. Even the sometimes suggested changes of variables are not here employed as, referring to [18], they may inappropriately stretch the grid of computation without making actual improvements.

SABO results are compared also with the perturbative method illustrated in [15] which is based on a series expansion and whose results depend on the number of terms considered in the truncated series and on a further choice of the perturbation parameter.

\section{Numerical results and comparisons}

\subsection{Constant parameters}

This example is taken from the paper [9] and it serves as a test and to compare the efficiency of SABO with other numerical methods.

With constant $r, d$ and $\sigma$, the problem (1)-(3) leads back to the standard Black-Scholes case whose solution is known in the closed-form (7).

- An up-and-out put option with the following data is considered:

Table 1. Up-and-out put option
data.
\begin{tabular}{|c|c|c|c|c|c|c|}
\hline$S_{u}$ & $t_{0}$ & $T$ & $E$ & $r$ & $d$ & $\sigma$ \\
\hline 2 & 0 & 1 & 1 & 0.1 & 0 & 0.25 \\
\hline
\end{tabular}

Denoting by $V_{F D}$ the Finite Difference approximation and by $V$ the exact solution in (7), the absolute errors reported in Table 2 are defined as

$$
\text { Err }:=\max _{i}\left|V_{F D}\left(\bar{S}_{i}, 0\right)-V\left(\bar{S}_{i}, 0\right)\right|
$$

over the same set of underlying asset values $\bar{S}_{i}=i \cdot E / 10=i \cdot 5$ for $i=1, \ldots, 9$ although the FD time-space grid is refined by the relation $\Delta t=\Delta S^{2}$.

Observe the rapid growth of CPU computation time w.r.t. the decrease of absolute error.

Choosing $\Delta t=T / 2^{n}$ for the evaluation of option price by SABO, the first column in Table 3 represents the evolution of the absolute error computed over the above set $\left\{\bar{S}_{i}\right\}_{i=1, \ldots, 9}$, the second one reports the CPU time spent in computing the numerical approximation of BIE solution as described in Section 3.3, the third one reports the CPU time spent in the post-processing as detailed in Section 3.4 and the fourth one the CPU time employed for the whole application of SABO.

Looking at Table 3 one can observe that SABO allows to achieve higher order of accuracy with lower computational costs w.r.t. FD. 


\section{Semi-Analytical method for the pricing of Barrier Options}

There is a worsening in CPU time spent in calculation in comparison to results appearing in [9] that is due to the use of Matlab ${ }^{\circledR}$ adaptive quadrature (with absolute error and relative error tolerances equal to $10^{-6}$ that could be weakened) instead of using ad hoc quadrature developed for the involved integrals. The reason of this questionable choice is that the idea of this article is to provide a user-friendly method for option pricing requiring no deep knowledge of quadrature techniques. However the method is still advantageous with respect to FD. Moreover, SABO is even more competitive when we have to compute the option value for only one underlying asset value because the CPU time spent in the post-processing decreases to about one twentieth of that actually written.

Table 2. Results obtained by FD with $\Delta S:=2^{-n} / 5$.

\begin{tabular}{c|c|c}
$n$ & Err & CPU time FD \\
\hline 1 & $2.2 \cdot 10^{-3}$ & $3.1 \cdot 10^{-2}$ \\
2 & $5.3 \cdot 10^{-4}$ & $7.8 \cdot 10^{-2}$ \\
3 & $1.3 \cdot 10^{-4}$ & $1.7 \cdot 10^{-1}$ \\
4 & $3.3 \cdot 10^{-5}$ & $1.6 \cdot 10^{+0}$ \\
5 & $8.2 \cdot 10^{-6}$ & $1.3 \cdot 10^{+2}$
\end{tabular}

Table 3. Results obtained by SABO with $\Delta t=T / 2^{n}$.

\begin{tabular}{c|c|c|c|c}
$n$ & Err & CPU time BIE & CPU time post-pro & CPU time SABO \\
\hline 2 & $1.9 \cdot 10^{-6}$ & $7.0 \cdot 10^{-1}$ & $1.6 \cdot 10^{+0}$ & $2.3 \cdot 10^{+0}$ \\
3 & $5.4 \cdot 10^{-7}$ & $1.5 \cdot 10^{+0}$ & $2.1 \cdot 10^{+0}$ & $3.6 \cdot 10^{+0}$ \\
4 & $2.3 \cdot 10^{-7}$ & $3.9 \cdot 10^{+0}$ & $3.1 \cdot 10^{+0}$ & $7.0 \cdot 10^{+0}$ \\
5 & $9.1 \cdot 10^{-8}$ & $1.1 \cdot 10^{+1}$ & $4.6 \cdot 10^{+0}$ & $1.6 \cdot 10^{+1}$ \\
6 & $3.2 \cdot 10^{-8}$ & $3.4 \cdot 10^{+1}$ & $8.4 \cdot 10^{+0}$ & $4.2 \cdot 10^{+1}$
\end{tabular}

In Fig. 1 from left to right, as a function of the underlying asset values $\left\{\bar{S}_{i}\right\}$, there are: the up-and-out put option price together with the payoff, the absolute errors made by FD with $\Delta S=0.00625$ and by SABO with $\Delta t=0.25$. As expected, FD has more troubles at the exercise price value $\bar{S}_{5}=E=1$ where the payoff function has a discontinuity in the first derivative, instead, SABO struggles with integration near the boundary point $S_{u}$.

Taking into account the approximation of $\boldsymbol{\Delta}$-greek, one can observe the advantage of using SABO instead of a finite difference approximation comparing the errors w.r.t. the available closed-formula (see [20]).

The evaluation of $\boldsymbol{\Delta}$-greek is compared among all the methods within the above set of underlying asset values $\left\{\bar{S}_{i}\right\}_{i=1, \ldots, 9}$. In Table 4 the $\boldsymbol{\Delta}$-greek is directly computed without the evaluation of option values following the 


\section{Guardasoni}
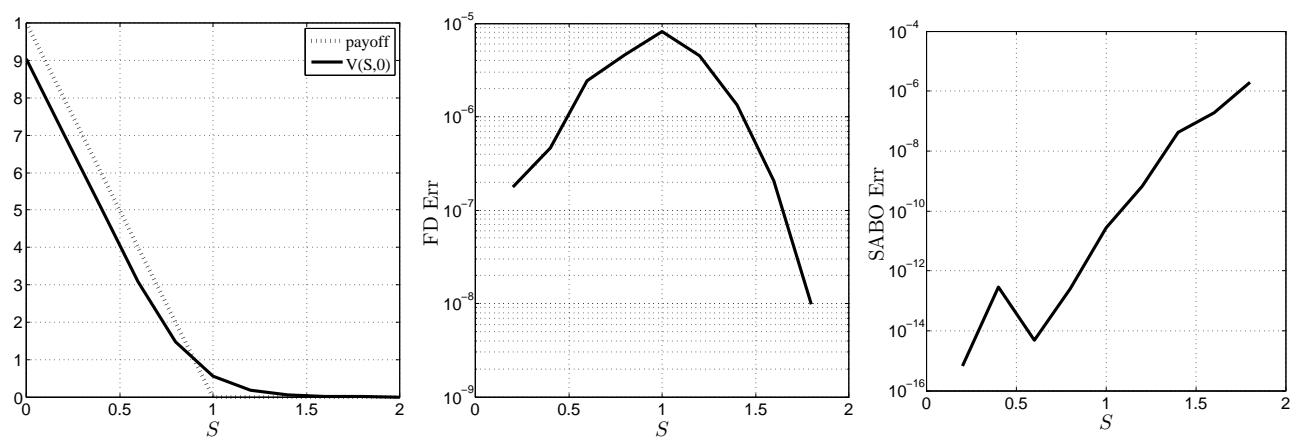

Figure 1. From left to right: up-and-out put option price, absolute error made by FD with $\Delta S=0.00625$ and by $\mathrm{SABO}$ with $\Delta t=0.25$.

algorithm suggested in Section 4 with computational times similar to those reported in Table 3.

Table 4. Results, concern-
ing $\Delta$-greek, obtained by SABO with
$\Delta t=T / 2^{n}$.
\begin{tabular}{c|c|c}
$n$ & Err & CPU time SABO \\
\hline 2 & $2.8 \cdot 10^{-5}$ & $4.2 \cdot 10^{+0}$ \\
3 & $4.4 \cdot 10^{-6}$ & $5.9 \cdot 10^{+0}$ \\
4 & $8.9 \cdot 10^{-7}$ & $1.0 \cdot 10^{+1}$ \\
5 & $4.9 \cdot 10^{-7}$ & $2.2 \cdot 10^{+1}$ \\
6 & $1.8 \cdot 10^{-7}$ & $5.3 \cdot 10^{+1}$
\end{tabular}

Differently, once option values have been computed by FD over a grid, $\boldsymbol{\Delta}$-greek can be approximated with negligible computational effort by the second order formula:

$$
\boldsymbol{\Delta}_{F D}\left(\bar{S}_{i}, 0\right) \approx \frac{V\left(\bar{S}_{i}+\Delta S, 0\right)-V\left(\bar{S}_{i}-\Delta S, 0\right)}{2 \Delta S}
$$

but with the absolute errors displayed in Table 5 that are, as expected, worse than those in Table 4, while being CPU time similar.

The exploitation of a superior order formula such as

$\boldsymbol{\Delta}_{F D}\left(\bar{S}_{i}, 0\right) \approx \frac{-V\left(\bar{S}_{i}+2 \Delta S, 0\right)+8 V\left(\bar{S}_{i}+\Delta S, 0\right)-8 V\left(\bar{S}_{i}-\Delta S, 0\right)+V\left(\bar{S}_{i}-2 \Delta S, 0\right)}{12 \Delta S}$ 


\section{Semi-Analytical method for the pricing of Barrier Options}

\begin{tabular}{|c|c|}
\hline & $\begin{array}{l}\text { lute errors } \\
\text { by a } 2 \text { nd } \\
\text { approx- } \\
\text { on formula } \\
\text {-greek, with } \\
=2^{-n} / 5 \text {. }\end{array}$ \\
\hline$n$ & Err \\
\hline 2 & $9.8 \cdot 10^{-3}$ \\
\hline 3 & $2.5 \cdot 10^{-3}$ \\
\hline 4 & $6.3 \cdot 10^{-4}$ \\
\hline J & $1.6 \cdot 10^{-4}$ \\
\hline 6 & $4.0 \cdot 10^{-5}$ \\
\hline
\end{tabular}

Table 6. Absolute errors made by a 4 th order approximation formula of $\Delta$-greek, with $\Delta S:=2^{-n} / 5: \operatorname{Err}_{F D}$ refers to the errors made by using FD option values and Err to the ones made by using closed-form option values.

is useless unless we consider values obtained by the closed formula (7) (as in Table 6) or by another method with greater accuracy than FD (but probably with more computational effort too) because, otherwise, the values themselves are affected by errors of the order of the FD method.

Finally in Fig. 2, from left to right, there are: the graph of the closedform $\Delta$-greek, the absolute error made by FD second order formula with $\Delta S=0.00625$ and by $\mathrm{SABO}$ with $\Delta t=0.25$.
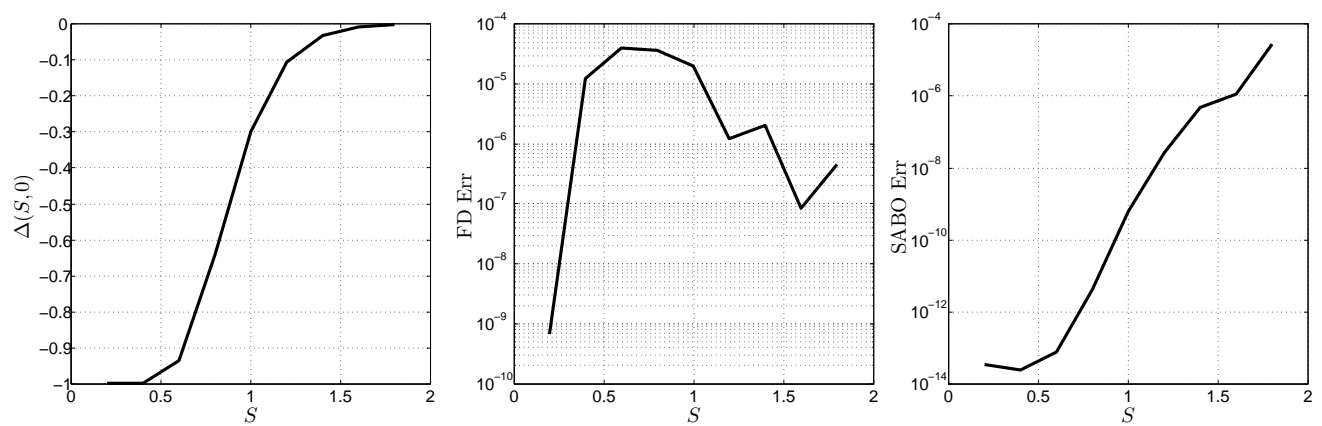

Figure 2. From left to right: up-and-out put option $\Delta$-greek, absolute error made by FD with $\Delta S=0.00625$ and by SABO with $\Delta t=0.25$.

\subsection{Time dependent $\sigma$}

- Piecewise constant $\sigma$

This example is taken from the paper [15] and the considered data for the up-and-out put option are those in Table 7.

The option price approximated by $\mathrm{SABO}, V_{S A B O}\left(S, t_{0}\right)$, is evaluated for two different values of strike price $E=101,103$. 


\section{Guardasoni}

Table 7. Up-and-out put option data taken from [15].

\begin{tabular}{|c|c|c|c|c|c|c|}
\hline$S_{u}$ & $t_{0}$ & $T$ & $S$ & $r$ & $d$ & \multicolumn{2}{|c|}{$\sigma(t)$} \\
\hline 101 & 0 & 0.5 & 100 & 0.03 & 0.02 & $\begin{cases}\sigma_{1}=0.105 & t<0.25 \\
\sigma_{2}=0.1147824 & 0.25 \leq t \leq T\end{cases}$ \\
\hline
\end{tabular}

Comparing our results to those in [15], we can immediately observe that the convergence of SABO strictly depends only on refining the discretization parameter $\Delta t=\left(T-t_{0}\right) / 2^{n}$ without the need to check assumptions on financial parameters or to decide the number of series expansion terms. The efficiency of SABO method can be checked in Tables 8 and 9, for $E=101$ and $E=103$ respectively. The reference solutions suggested in [15] $(\widetilde{V}(100,0)=0.8944$ and $\widetilde{V}(100,0)=1.0884$, respectively) are obtained by a not specified finite difference method and truncated to $10^{-4}$ order of accuracy, so we have put on the right the results obtained by FD method.

Table 8. Strike $E=101$. Reference Value in $[15]: \widetilde{V}(100,0)=0.8944$. On the left results obtained by $\mathrm{SABO}$ with $\Delta t=T / 2^{n}$. On the right results obtained by FD with $\Delta t=\Delta x^{2}$ and $\Delta x=0.25 / 2^{n}$.

\begin{tabular}{|c|c|c|c|c|c|}
\hline$n$ & $V_{S A B O}(100,0)$ & CPU time (s) & $n$ & $V_{F D}(100,0)$ & CPU time (s) \\
\hline 2 & 0.89178 & $1.0 \cdot 10^{+0}$ & & & $1.0 \cdot 10^{-1}$ \\
\hline 3 & 0.89373 & $2.0 \cdot 10^{+0}$ & 0 & 0.89584 & $1.0 \cdot 10+0$ \\
\hline 4 & 0.89419 & $4.5 \cdot 10^{+0}$ & 1 & 0.89474 & $2.1 \cdot 10^{+0}$ \\
\hline 5 & 0.89433 & $1.3 \cdot 10^{+1}$ & 2 & 0.89447 & $3.4 \cdot 10^{+1}$ \\
\hline 6 & 0.89436 & $3.9 \cdot 10^{+1}$ & 3 & 0.89440 & $3.4 \cdot 10^{+2}$ \\
\hline 7 & 0.89437 & $1.2 \cdot 10^{+2}$ & 4 & 0.89438 & $3.4 \cdot 10^{+3}$ \\
\hline
\end{tabular}

Table 9. Strike $E=103$. Reference Value in $[15]: \widetilde{V}(100,0)=1.0884$. On the left results obtained by $\mathrm{SABO}$ with $\Delta t=T / 2^{n}$. On the right results obtained by FD with $\Delta t=\Delta x^{2}$ and $\Delta x=0.25 / 2^{n}$.

\begin{tabular}{l|c|c}
$n$ & $V_{S A B O}(100,0)$ & CPU time $(\mathrm{s})$ \\
\hline 2 & 1.08163 & $1.0 \cdot 10^{+0}$ \\
3 & 1.08634 & $1.9 \cdot 10^{+0}$ \\
4 & 1.08787 & $4.5 \cdot 10^{+0}$ \\
5 & 1.08828 & $1.2 \cdot 10^{+1}$ \\
6 & 1.08839 & $3.7 \cdot 10^{+1}$ \\
7 & 1.08842 & $1.2 \cdot 10^{+2}$
\end{tabular}

\begin{tabular}{c|c|c}
$n$ & $V_{F D}(100,0)$ & CPU time $(\mathrm{s})$ \\
\hline 0 & 1.10233 & $1.6 \cdot 10^{-1}$ \\
1 & 1.09175 & $2.4 \cdot 10^{+0}$ \\
2 & 1.08926 & $3.5 \cdot 10^{+1}$ \\
3 & 1.08864 & $3.7 \cdot 10^{+2}$ \\
4 & 1.08849 & $3.7 \cdot 10^{+3}$
\end{tabular}

If $E=201$ then the solution is forced to noticeably change its slope as observable in Fig. 3 and this swing is acknowledged by the $\Delta$-greek computed as in formula (25)

The change in volatility influences the evolution of the solution: the price of the option from maturity $T=0.5$ until time $t=0.25$ is that of an Eu- 
Semi-Analytical method for the pricing of Barrier Options
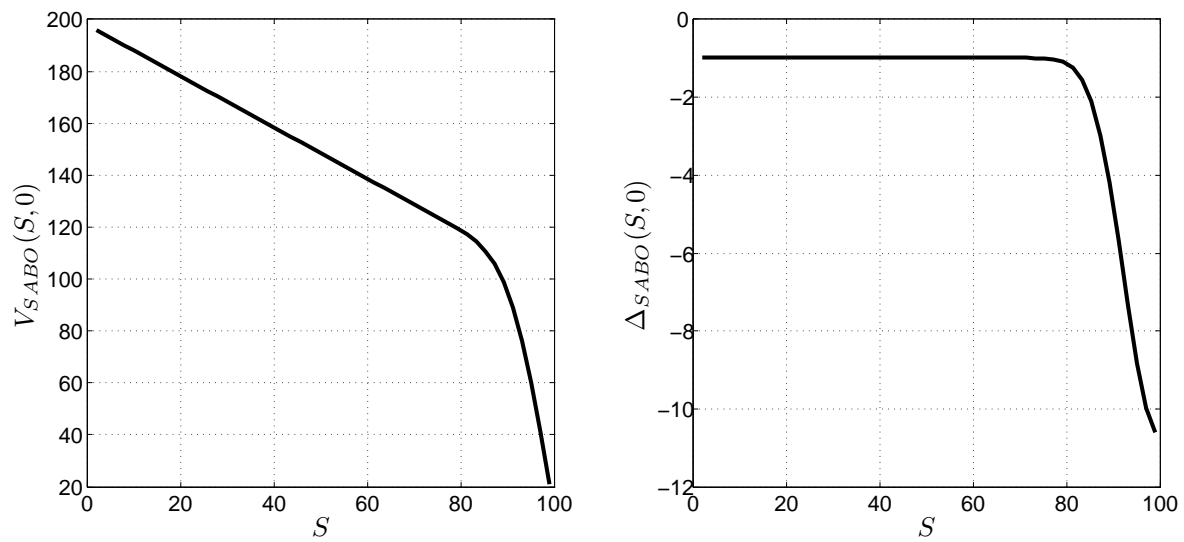

Figure 3. Up-and-out put option with parameters in Table 7 and exercise price $E=201$. On the left: approximated value obtained by $\mathrm{SABO}$ with $\Delta t=0.0625$. On the right: approximated value of $\boldsymbol{\Delta}$-greek obtained by SABO with $\Delta t=0.0625$.

ropean put up-and-out option $V_{2}$ with constant volatility equal to $\sigma_{2}$ then it moves towards the price of an European put up-and-out option $V_{1}$ with constant volatility equal to $\sigma_{1}$. This evolution is highlighted at underlying asset value $S=80$. In Fig. 4 on the left, the approximated solution $V_{S A B O}(80, t)$ (obtained with $\left.\Delta t=0.0078125\right)$ in the time interval $[0,0.25]$ has clearly an intermediate value between $V_{1}(80, t)$ and $V_{2}(80, t)$ but, on display, the three solutions are almost overlapped in the half interval of the expiry. Looking at Fig. 4 on the right, the difference between $V_{1}(80, t)$ and $V_{S A B O}(80, t)$ respectively and $V_{2}(80, t)$ emphasizes the expected behavior also in the interval $[0.25,0.5]$ and in particular the sudden effects due to the volatility change at $t=0.25$.

- Time-continuous $\sigma$

The data of this example are taken from the paper [15] and again applied to the case of an up-and-out put option:

Table 10. Up-and-out put option data taken from [15].

\begin{tabular}{|c|c|c|c|c|c|c|}
\hline$S_{u}$ & $E$ & $S$ & $t_{0}$ & $r$ & $d$ & $\sigma(t)$ \\
\hline 70 & 50 & 50 & 0 & 0.1 & 0.05 & $\sigma^{2}=0.03+0.02(T-t)$ \\
\hline
\end{tabular}

In Fig. 5, the approximated solution obtained by SABO with $\Delta t=$ $\left(T-t_{0}\right) / 8$ is plotted for different values of expiry $T=0.25,0.5,0.75,1$. As foreseeable, the closer the expiry the nearer the option value to the payoff function.

Looking at Tables 11 and 12, one can appreciate, at a randomly chosen 


\section{Guardasoni}
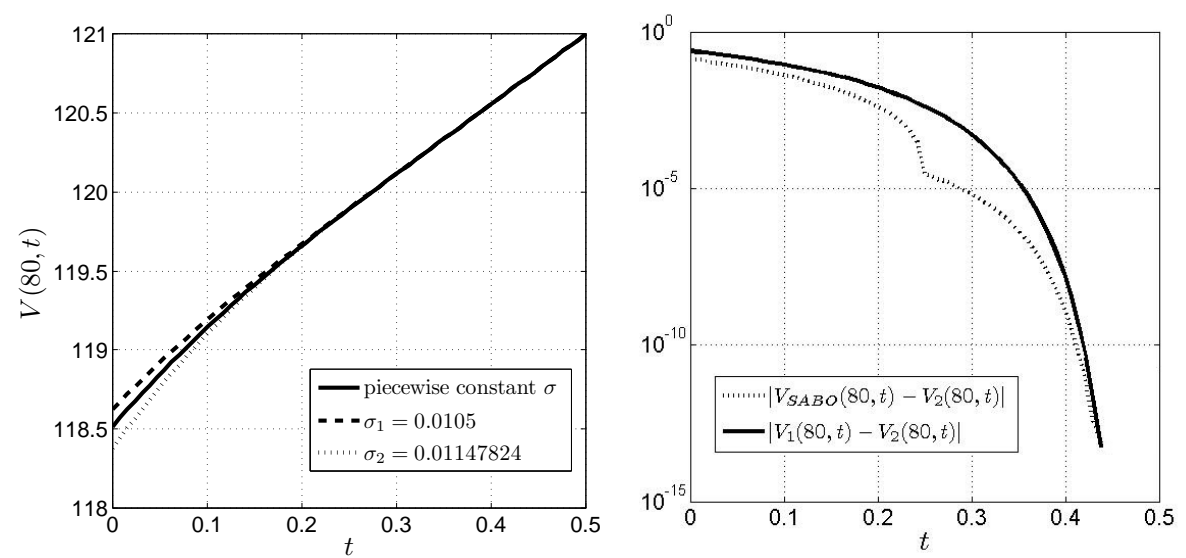

Figure 4. On the left: at $S=80$, approximated option value with piecewise constant volatility reported in Table 7 and closed form option values with constant volatility equal to $\sigma_{1}$ and $\sigma_{2}$ respectively. On the right: distance from $V_{2}(80, t)$ of $V_{S A B O}(80, t)$ and $V_{2}(80, t)$.

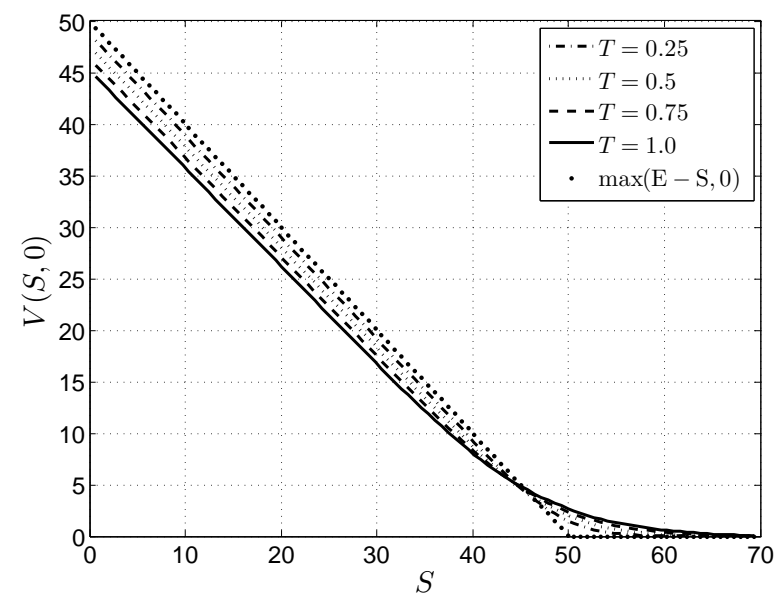

Figure 5. SABO approximate solutions for different values of expiry time.

point, that results by SABO (in comparison with those obtained by FD method) are even more accurate than in the case of the previous example because of the better regularity of the volatility function $\sigma$. Very good accuracy is achieved spending little CPU time and, also in this case, it strictly depends only on refining the discretization parameter $\Delta t=(T-$ $\left.t_{0}\right) / 2^{n}$ and it does not need checking assumptions on data or tuning further parameters (as, on the contrary, happens in [15]). 


\section{Semi-Analytical method for the pricing of Barrier Options}

Table 11. Approximate option values obtained by FD method at $S=50$ and $t=0$ with $\Delta S=0.25 / 2^{n}$ and $\Delta t=\Delta x^{2}$.

\begin{tabular}{c|c|c|c|c|c|c|c|c}
$n$ & $T=1$ & CPU time $(\mathrm{s})$ & $T=0.75$ & CPU time & $T=0.5$ & CPU time & $T=0.25$ & CPU time \\
\hline 0 & 2.64610 & $7.8 \cdot 10^{-2}$ & 2.34161 & $7.8 \cdot 10^{-2}$ & 1.96357 & $9.4 \cdot 10^{-2}$ & 1.43428 & $6.3 \cdot 10^{-2}$ \\
1 & 2.64919 & $2.6 \cdot 10^{+0}$ & 2.34889 & $1.9 \cdot 10^{+0}$ & 1.97826 & $1.2 \cdot 10^{+0}$ & 1.46420 & $6.3 \cdot 10^{-1}$ \\
2 & 2.64991 & $4.4 \cdot 10^{+1}$ & 2.35066 & $3.3 \cdot 10^{+1}$ & 1.98191 & $2.1 \cdot 10^{+1}$ & 1.47175 & $1.0 \cdot 10^{+1}$ \\
3 & 2.65008 & $6.0 \cdot 10^{+2}$ & 2.35110 & $4.2 \cdot 10^{+2}$ & 1.98282 & $2.8 \cdot 10^{+2}$ & 1.47364 & $1.4 \cdot 10^{+2}$
\end{tabular}

Table 12. Approximate option values obtained by SABO at $S=50$ and $t=0$ with $\Delta t=\left(T-t_{0}\right) / 2^{n}$.

\begin{tabular}{c|c|c|c|c|c}
$n$ & $T=1$ & $T=0.75$ & $T=0.5$ & $T=0.25$ & CPU time $(\mathrm{s})$ \\
\hline 2 & 2.65021 & 2.35125 & 1.98312 & 1.47427 & $9.4 \cdot 10^{-1}$ \\
3 & 2.65017 & 2.35125 & 1.98312 & 1.47427 & $1.9 \cdot 10^{+0}$ \\
4 & 2.65015 & 2.35125 & 1.98312 & 1.47427 & $4.2 \cdot 10^{+0}$ \\
5 & 2.65015 & 2.35125 & 1.98312 & 1.47427 & $1.2 \cdot 10^{+1}$
\end{tabular}

\subsection{Time dependent interest rate}

- At first, the up-and-out put option is evaluated with the following data:

Table 13. Up-and-out put option data.

\begin{tabular}{|c|c|c|c|c|c|c|c|}
\hline$S_{u}$ & $t_{0}$ & $T$ & $S$ & $E$ & $r(t)$ & $d$ & $\sigma$ \\
\hline 101 & 0 & 1 & 50 & 50 & $\left\{\begin{array}{l}r_{1}=0.01 t<0.25 \\
r_{2}=0.030 .25 \leq t \leq T\end{array}\right.$ & 0.05 & 0.105 \\
\hline
\end{tabular}

In Fig. 6 the time-behavior of the approximated solution $V$ at $S=50$ is depicted together with its $\boldsymbol{\Theta}$-greek. Both are obtained by SABO, inserting the BIE solution in (23) and (31), respectively. Due to the discontinuity of the interest rate function at $t=0.25$, we expect the solution to be just continuous in time variable and the time-derivative to have a jump at the same time instant. As viewable in Fig. 6 in the center, SABO allows to precisely catch this jump because the expression of the $\boldsymbol{\Theta}$-greek is known in a piecewise closed-form (except that the BIE solution $\frac{\partial u}{\partial y}(U, s)$ has to be numerically computed): the discontinuity is highlighted in the second term by the multiplication of $V$ by $\bar{r}$. On the contrary, the application of a finite difference approximation of time-derivative, analogous to (34) in time, to accurate $\mathrm{SABO}$ option values, is based on the hypothesis of $\mathcal{C}^{1}$-regularity of the solution and therefore it softens the discontinuity as focused in Fig. 6 on the right.

- Choosing the barrier smaller than the strike price and the reference asset value near the barrier, the contribution of the boundary integral term, i.e. the second term in the r.h.s. of representation formula (13), is stronger 


\section{Guardasoni}
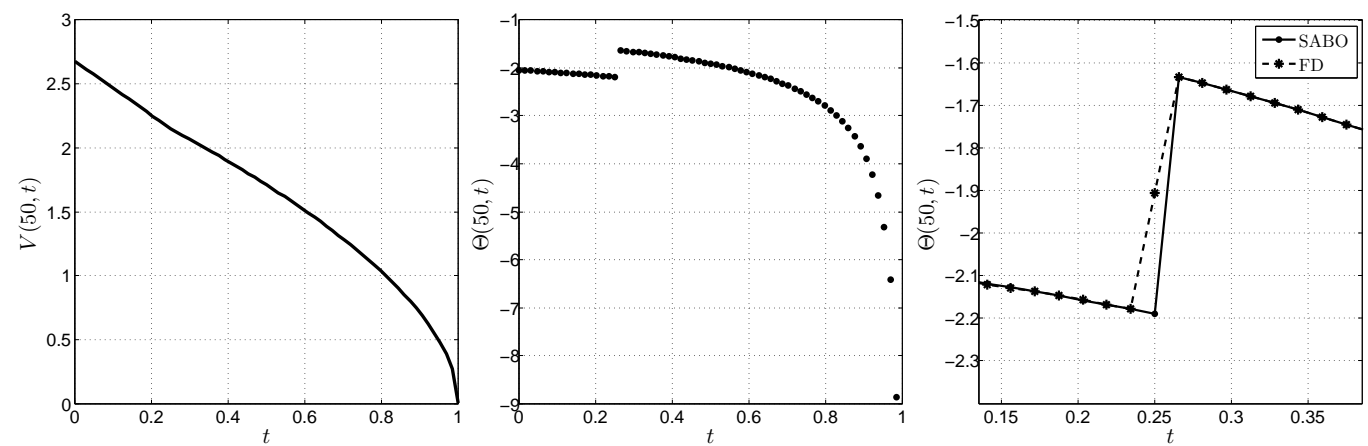

Figure 6. On the left: at $S=50$, evolution in time of approximated option value with piecewise constant interest rate reported in Table 13 and computed by SABO with $\Delta t=$ 0.015625. In the center: its $\Theta$-greek. On the right: zoom nearby the time instant $t=0.25$ of the $\boldsymbol{\Theta}$-greek computed by SABO and finite difference method.

so that the barrier option price moves significantly away from the vanilla option price really near the barrier and the computation of greeks gives more troubles (look at Fig. 7).

Table 14. Up-and-out put option data.

\begin{tabular}{|c|c|c|c|c|c|c|c|}
\hline$S_{u}$ & $t_{0}$ & $T$ & $S$ & $E$ & $r(t)$ & $d$ & $\sigma$ \\
\hline 40 & 0 & 1 & 35 & 50 & $\left\{\begin{array}{l}r_{1}=0.01 t<0.25 \\
r_{2}=0.030 .25 \leq t \leq T\end{array}\right.$ & 0.05 & 0.105 \\
\hline
\end{tabular}
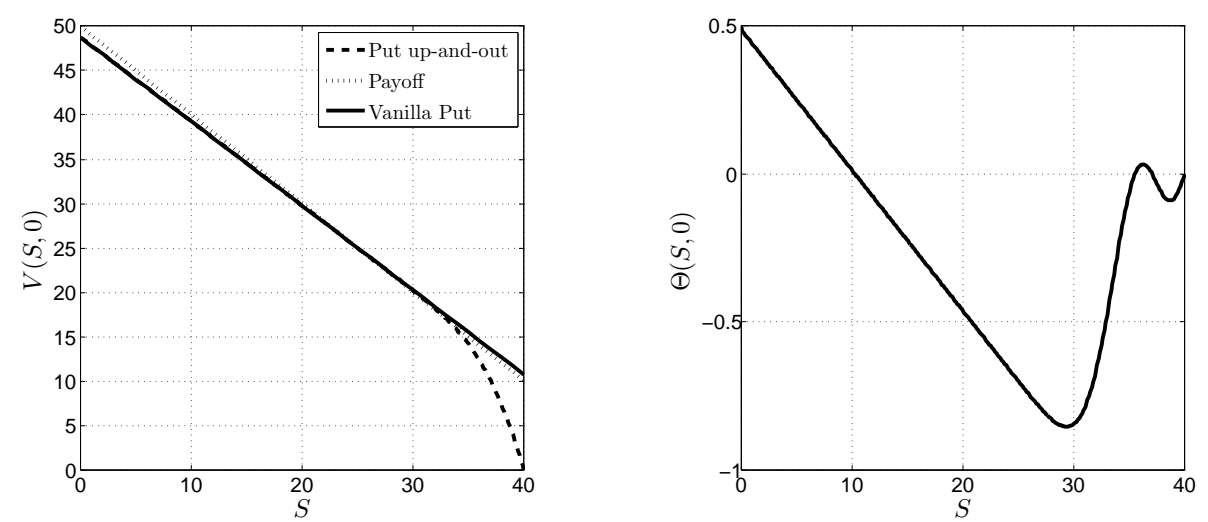

Figure 7. Approximated option value with data of Table 14 (on the left) and $\Theta$-greek (on the right) equivalently computed by SABO or FD at time instant $t=0$.

This situation stresses the computation of both SABO and FD method but, 


\section{Semi-Analytical method for the pricing of Barrier Options}

as viewable in Table 15 at $S=35$ and $t=0$, SABO gets a superior order of accuracy in evaluation of both the option price and the $\boldsymbol{\Theta}$-greek with remarkable computational saving w.r.t. FD method.

Table 15. Put up-and-out option price at $S=35$ and $t=0$ obtained by SABO with $\Delta t=T / 2^{n}$ (on the left) and by FD with $\Delta t=\Delta x^{2}$ and $\Delta x=0.25 / 2^{n}$ (on the right).

\begin{tabular}{|c|c|c|c|c|c|c|c|}
\hline$n$ & $V_{S A B O}(35,0)$ & $\boldsymbol{\Theta}_{S A B O}(35,0)$ & CPU time & $n$ & $V_{F D}(35,0)$ & $\boldsymbol{\Theta}_{F D}(35,0)$ & CPU time \\
\hline 4 & 14.13354 & -0.06753 & $1.7 \cdot 10^{+0}$ & 0 & 14.24680 & -0.03076 & $3.1 \cdot 10^{-2}$ \\
\hline 8 & 14.13236 & -0.07581 & $3.1 \cdot 10^{+0}$ & 1 & 14.16124 & -0.06392 & $5.0 \cdot 10^{-1}$ \\
\hline 16 & 14.13188 & -0.07724 & $7.1 \cdot 10^{+0}$ & 2 & 14.13902 & -0.07417 & $1.3 \cdot 10^{+1}$ \\
\hline 32 & 14.13168 & -0.07760 & $1.9 \cdot 10^{+1}$ & 3 & 14.13341 & -0.07686 & $2.0 \cdot 10^{+2}$ \\
\hline 64 & 14.13160 & -0.07771 & $6.0 \cdot 10^{+1}$ & 4 & 14.13201 & -0.07754 & $2.8 \cdot 10^{+3}$ \\
\hline
\end{tabular}

\section{Conclusions and developments}

A new method for pricing barrier option (SABO) is illustrated. The method was already introduced for the Black-Scholes model with timeconstant parameters and for Heston-Bates model (see [9] and [10] respectively). Its adaptation to the Black-Scholes model with time-dependent parameters is here proposed with several numerical examples and the computation of the Greeks. Furthermore, some ready to use Matlab codes described in Appendix II are downloadable at ????.

The main advantages of SABO in comparison with other numerical methods are: the high accuracy, the implicit satisfaction of the far-field behavior of the solution, the low discretization costs that imply high efficiency and the easy computation of Greeks. We think that the method could have a considerable range of application that in these last years we started investigating. In particular we expect to be able to soon apply the method to Asian Options too.

\section{Acknowledgements}

We would like to thank University of Parma for the concession of a grant addressed to the research on this topic.

\section{Bibliography}

\section{REFERENCES}

1. F. Black and M. Scholes, The pricing of options and corporate liabilities, The Journal of Political Economy, vol. 81, no. 3, pp. 637-654, 1973.

2. M. Danilo and V. Spokoinyi, Estimation of time dependent volatility via local change point analysis, Annals of Statistics, vol. 32, no. 2, p. $577 i \iota \frac{1}{2} 602,2004$. 


\section{Guardasoni}

3. L. Goldentayer, F. Klebaner, and R. Liptser, Tracking volatility, Problems of Information Transmission, vol. 41, no. 3, pp. 212-229, 2005.

4. S. Heston, A closed-form solution for options with stochastic volatility with applications to bond and currency options, Review of Financial Studies, vol. 6, pp. 327-343, 1993.

5. D. Bates, Jumps and stochastic volatility: Exchange rate processes implicit in deutsche mark options, The Review of Financial Studies, vol. 9, no. 1, pp. 69-107, 1996.

6. L. Feng and V. Linetsky, Pricing options in jump-diffusion models: an extrapolation approach, Oper. Res., vol. 56, no. 2, pp. 304-325, 2008.

7. F. Fang and C. W. Oosterlee, A Fourier-based valuation method for Bermudan and barrier options under Heston's model, SIAM J. Financial Math., vol. 2, no. 1, pp. 439-463, 2011.

8. G. Fusai, G. Germano, and D. Marazzina, Spitzer identity, WienerHopf factorization and pricing of discretely monitored exotic options, European J. Oper. Res., vol. 251, no. 1, pp. 124-134, 2016.

9. C. Guardasoni and S. Sanfelici, A boundary element approach to barrier option pricing in Black-Scholes framework, Int. J. Comput. Math., vol. 93, no. 4, pp. 696-722, 2016.

10. C. Guardasoni and S. Sanfelici, Fast Numerical Pricing of Barrier Options under Stochastic Volatility and Jumps, SIAM J. Appl. Math., vol. 76, no. 1, pp. 27-57, 2016.

11. F. Rapisarda, Pricing barriers on underlyings with time-dependent parameters, tech. rep., Banca IMI, 2005.

12. G. Dorfleitner, P. Schneider, K. Hawlitschek, and A. Buch, Pricing options with Green's functions when volatility, interest rate and barriers depend on time, Quantitative Finance, vol. 8, no. 2, pp. 119-133, 2008.

13. C. Lo, H. Lee, and C. Hui, A simple approach for pricing barrier options with time-dependent parameters, Quantitative Finance, vol. 3, pp. 98107, 2003.

14. C. Lo and C. Hui, Valuing double barrier options with time-dependent parameters by fourier series expansion, International Journal of Applied Mathematics, vol. 36, no. 1 .

15. L. Fatone, M. Recchioni, and F. Zirilli, A perturbative formula to price barrier options with time-dependent parameters in the Black and Scholes world, Journal of Risk, vol. 10, pp. 131-146, Winter 2007/2008.

16. L. Ballestra and G. Pacelli, A very fast and accurate boundary element method for options with moving barrier and time-dependent rebate, Applied Numerical Mathematics, vol. 77, pp. 1 - 15, 2014.

17. F. Rapisarda, Barrier options on underlyings with time-dependent parameters: a perturbation expansion approach, tech. rep., Banca IMI, 


\section{Semi-Analytical method for the pricing of Barrier Options}

2005.

18. S. Sanfelici, Comparison of numerical methods for the approximation of option price, tech. rep., University of Parma, 2001.

19. P. Wilmott, J. Dewynne, and S. Howison, Option Pricing: Mathematical models and computation. Oxford Financial Press, 1993.

20. E. Reiner and M. Rubinstein, Breaking down the barriers, Risk, vol. 4, pp. 28-35, 1991.

21. A. Friedman, Partial Differential Equations of Parabolic type. Englewood Cliffs, N.Y.: Prentice-Hall Inc, 1964.

22. A. Friedman, Stochastic Differential Equations and Applications. Academic Press, 1975.

23. A. Mijatović, Local time and the pricing of time-dependent barrier options, Finance and Stochastics, vol. 14, no. 1, pp. 13-48, 2010.

24. A. Aimi, M. Diligenti, and C. Guardasoni, Numerical integration schemes for space-time hypersingular integrals in energetic Galerkin BEM, Numerical Algorithms, vol. 55, no. 2, pp. 145-170, 2010.

25. P. Wilmott, Derivatives: the theory and practice of financial engineering. John Wiley and Sons, 2000.

26. R. U. Seydel, Tools for computational finance. Universitext, SpringerVerlag, Berlin, fourth ed., 2009.

27. R. Rannacher, Finite element solution of diffusion problems with irregular data, Numer. Math., vol. 43, no. 2, pp. 309-327, 1984.

28. G. Fusai, S. Sanfelici, and A. Tagliani, Practical Problems in the Numerical Solution of PDE's in Finance, Rendiconti per gli Studi Economici Quantitativi, pp. 105-132, 2002.

29. M. Giles and R.Carter, Convergence analysis of Crank-Nicolson and Rannacher time-marching, J. Comput. Finance, vol. 9, no. 4, pp. 89$112,2006$.

30. D. Pooley, K. Vetzal, and P. Forsyth, Convergence remedies for nonsmooth payoffs in option pricing, Journal of Computational Finance, vol. 6, no. 4, pp. 25-40, 2003.

31. R. Zvan, K. R. Vetzal, and P. A. Forsyth, PDE methods for pricing barrier options, J. Econom. Dynam. Control, vol. 24, no. 11-12, pp. 1563$1590,2000$.

\section{Appendix I: Put up-and-in}

Consider an up-and-in barrier put option with European exercise style: it is an exotic option that gets into existence with the value of an European put option without barriers $P$ (as defined in (8)) when the price of the underlying asset grows up enough to breach an assigned upper barrier 


\section{Guardasoni}

before the expiry date. The differential problem that models this option is analogous to (6) and it is

(35)

$$
\left\{\begin{array}{lr}
\frac{\partial u}{\partial \tau}(x, \tau)-\frac{\bar{\sigma}^{2}(\tau)}{2} \frac{\partial^{2} u}{\partial x^{2}}(x, \tau)-\left(\bar{r}-\frac{\bar{\sigma}^{2}}{2}-\bar{d}\right)(\tau) \frac{\partial u}{\partial x}(x, \tau)=0 & \\
x(x, 0)=0=: u_{0}(x) & x=(-\infty, U), \tau \in\left(0, T-t_{0}\right] \\
\lim _{x \rightarrow-\infty} u(x, \tau)=0 & x \in \Omega\left[0, T-t_{0}\right] \\
u(U, \tau)=P\left(S_{u}, t\right) & U:=\log \left(S_{u}\right), \tau \in\left[0, T-t_{0}\right]
\end{array}\right.
$$

and the domain of the problem is again $(-\infty, U) \times\left(0, T-t_{0}\right]$ because at $S \geq S_{u}$ the option takes the value of an European put option without barriers.

$\mathrm{SABO}$ is deduced from steps in Sec. 3 and it evolves as follows.

The integral representation formula in the domain of definition of the differential problem

The integral representation formula is still Eq. (13) because the problem has the same differential operator and it is defined over the same domain: $\forall(x, \tau) \in(-\infty, U) \times\left(0, T-t_{0}\right]$

$$
u(x, \tau):=\int_{-\infty}^{U} u_{0}(y) G(y, 0 ; x, \tau) d y+\int_{0}^{\tau} \frac{\bar{\sigma}^{2}(s)}{2} G(U, s ; x, \tau) \frac{\partial u}{\partial y}(U, s) d s,
$$

nevertheless, as the initial condition is null, it reduces to

$$
u(x, \tau):=\int_{0}^{\tau} \frac{\bar{\sigma}^{2}(s)}{2} G(U, s ; x, \tau) \frac{\partial u}{\partial y}(U, s) d s .
$$

The boundary integral equation (BIE)

The integral formula (37) is the analytical solution of problem (35) for $(x, \tau) \in \Omega \times\left(0, T-t_{0}\right]$ but it cannot be evaluated analytically since the function $\frac{\partial u}{\partial y}(U, s)$ is not known explicitly.

However, letting $x \rightarrow U$ in (13) and applying the boundary condition at the barrier, we obtain the BIE

$$
P\left(S_{u}, t\right)=\int_{-\infty}^{+\infty} \max \left(E-e^{y}, 0\right) G(y, 0 ; U, \tau) d y=\int_{0}^{\tau} \frac{\bar{\sigma}^{2}(s)}{2} \frac{\partial u}{\partial y}(U, s) G(U, s ; U, \tau) d s .
$$

whose sole unknown is the function $\frac{\partial u}{\partial y}(U, s)$.

Again, (38) is numerically solved at the boundary as in Sec.3.3 getting 


\section{Semi-Analytical method for the pricing of Barrier Options}

$\frac{\partial u}{\partial y}(U, s)$ and then the solution $u$ is recovered at the desired points of the whole domain by inserting it in the representation formula (37).

\section{Appendix II: Matlab ${ }^{\circledR}$ Codes}

The downloadable Matlab ${ }^{\circledR}$ codes (available as Supplemental Material of the present paper) allow the user to compute by SABO the value of a PUT option with UP-and-OUT barrier with the parameters shown in Table 10 related to the case of time-continuous $\sigma$ described in Section 6.2. 